\title{
Migration to Cloud Computing: A Risk Homeostasis Methodology
}

\author{
Hamse Yuusuf \\ University of South Wales \\ Newport \\ United Kingdom
}

\author{
Christopher Tubb \\ University of South Wales \\ Newport \\ United Kingdom
}

\begin{abstract}
In recent years virtualization has gained significant momentum. Organisations are looking at this technology seriously because of potential savings and of scalability advantage. According to Gartner's survey in early 2010 [1] of 1600 CIOs around the world, virtualization and cloud computing was on top of their IT investment list. This interest has also resulted in slew of products and services from existing IT players as well as new comers which promise to offer many solutions to pave the path towards migrating to virtual environment. As organizations start investing in virtualization, they look towards their current IT setup and in an attempt to identify the best way they can take advantage of what cloud has to offer. For a given enterprises, getting on to cloud might be a complete new start from scratch; a limited deployment of new applications or migration of part of existing applications integrating backwards with onpremise applications. To take advantage of the virtualization, enterprise will need to define their short and long term cloud strategy. There is a need to consider factors specific to their businesses and determine their requirements, risks and benefits. Proper investigation by the enterprise will give insight in to the benefits and specific strategy they need to follow to gain the said benefits from cloud computing. This paper analyses cloud benefits, risks and proposes specific migration methodology which enterprises can adopt to make sure the migration and integration between onpremise and cloud happens with minimal disruption to business and results in maximum sustainable cost benefit.
\end{abstract}

\section{Keywords}

Cloud Computing, Cloud Migration, Cloud Risks.

\section{INTRODUCTION}

The maturity of resource virtualization and Grid Computing technologies [1] have led to the emergence of cloud computing. Concepts such as virtual organizations and services exposed via transparent interfaces have initially been explored in Grids [2]. Cloud Computing combines these concepts with new virtualization techniques and business models to enable on-demand access to a shared pool of configurable resources (e.g., servers, storage, applications and services) that can be rapidly provisioned and released with minimal management effort. In this way, Cloud computing provides a new means for delivering IT services, based on cost-efficient, scalable and on-demand provisioning guided by the users requirements. Exploiting the flexibility offered by virtualization, IT elements are exposed as a set of services (or even resources) that can be reserved by users for a specific timeframe.
Currently, single users, companies, and institutions deploy dynamic computing resources on clouds to execute their applications, exploring the advantages offered by the different levels of virtualization, including cost reduction [3] and reliability support [4]. The commercial model explored in clouds differs from traditional IT commercialization by demanding smaller up-front investment. Traditionally, a user buys a software or hardware, and then uses it respecting the conditions imposed by contractual agreements (e.g., number of licenses or number of allowed executions). Cloud Computing proposes the on-demand availability of different virtualized services that can be rented, accessed, customized, and exploited by users in a pay-as-you-go manner.

With migrating to cloud computing, both users and providers can explore advantages such as: users are not locked into a single IT solution or provider; software and hardware licenses costs are diluted and distributed between providers and users; providers can operate close to the maximum computing capacity and, at same time, maximize revenues; IT administration costs are also proportionally shared by users and providers.

On the other hand, in spite of the numerous opportunities that cloud computing provides, it introduces new challenges that needs to be understood to complete a successful migration. These issues affect IT as well as the businesses. However, since most cloud computing migration projects are driven by the IT, these problems are not effectively addressed. Hence, there is a need for a process model that provides a framework to allow IT and businesses to collaborate effectively to tackle these challenges. Finally, this paper presents the broad methodology for migration to cloud and discusses with enterprise managers involved in migrating to cloud. There are various ways to slice and approach the migration - but all should take in to consideration the business processes, architecture of existing systems, architecture of available services, interoperability between on-premise and virtual environment, maturity of virtualization technology and standards, short and long term cost savings, sustainability, data/security/regulation, user adoption, available Service Level Agreements (SLAs) and business criticality.

\section{BENEFITS IN CLOUD}

There are many benefits of cloud computing but, also, some accompanying caveats. As with any physical system, cloud computation must operate within physical boundary parameters.

The cloud offers the ability to provision massive amounts of computing power and storage, but these quantities are not infinite [7]. Therefore, cloud users might have to fi t their applications into one set of resource usage categories defined by the cloud provider. 
Cloud computational resources can be scaled up and down on demand and paid for on a metered usage basis [8]. This ability provides tremendous advantages for clients in that they do not have to maintain internal computing systems designed for peak loads that may occur only a small percentage of the time. The cloud paradigm also supports innovation in that a variety of new, advanced applications can be used in an affordable manner while reducing the total cost of ownership. Some applications that are of long duration and have stable computational requirements might be better served by inhouse or leased computers and storage than by paying cloud fees over a long period of time. These options have to be evaluated on a case-by-case basis. There are four primary benefits that cloud computing promises to enterprise customers: Simplicity, Scalability, Agility and Costs. They can reduce and simplify their cost structure. They can leverage the elasticity of cloud computing to make their business more agile and they can take advantage of the fact that they have outsourced some of their IT focus by dedicating their freed resources to activities that improve their core competencies.

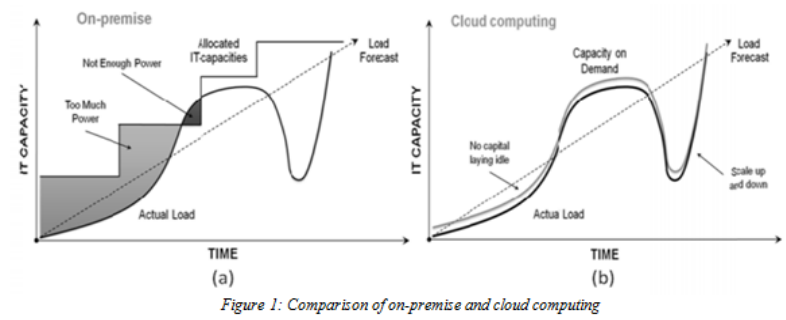

\subsection{Simplicity and Scalability}

The whole process of infrastructure provisioning and running applications is simple in Cloud space. Cloud offers simple provisioning, ramp-up and decommissioning which makes it easier for businesses to get better turnaround and test their ideas at a much cheaper and faster pace. Cloud computing provides the means, within limits, for a client to rapidly provision computational resources to meet increases or decreases in demand [9]. In many instances, organizations require large amounts of storage capacity for critical data, and this need can be accommodated by the cloud provider. This approach provides an alternative to inefficient in-house systems that have to be designed for peak load but run at only partial capacity most of the time [10]. Cloud scalability provides for remote optimization so that computing resources are organized for maximum cost-benefit. Because the cloud provider operates on a utility model, the client organization has to pay only for the resources it is using at the time. However, the cloud provider must provide some type of resource limits on customers to protect against extreme demands and ensure that there is enough capacity to serve all of the cloud clients.

\subsection{Agility}

Cloud enables organizations to be agile with their IT infrastructure and software needs by adding, removing IT resources on demand due to elasticity and use of shared resources inherent in the cloud implementations. In cloud, scalability, flexibility and elasticity are service attributes that positively contribute to business process value by reducing cycle times and accommodating volume fluctuations. Also In a competitive environment where rapid evaluation and development of new approaches is critical, the cloud offers the means to use powerful computational resources in a short time frame and large amounts of storage without requiring sizeable initial investments in hardware, software, and personnel. This rapid provisioning can be accomplished at relatively small cost and offers the client access to advanced technologies that are constantly being acquired by the cloud provider. Improved delivery of services obtained by rapid cloud provisioning improves time to market and market growth.

\subsection{Cost}

The cloud paradigm, in general, is a basis for cost savings because capability and resources can be paid for incrementally without the need for large investments in computing infrastructure. This model is especially true for adding storage costs for large database applications.

Therefore, capital costs are reduced and replaced by manageable, scalable operating expenses. Conversely, there might be some instances, particularly for long term, stable computing configuration usage, where cloud computation might not have a cost advantage over using ones internal resources or directly leasing equipment. For example, if the volume of data storage and computational resources required are essentially constant and there is no need for rapid provisioning and flexibility, organizations local computational capabilities might be more cost effective than using a cloud. Another factor to consider in choosing the cloud is that client organizational support and maintenance costs are reduced dramatically because these expenses are transferred to the cloud provider, including 24/7 support. The need for highly trained and expensive IT personnel is also reduced. Resources are used more efficiently in cloud computing, resulting in substantial support and energy cost savings. In general, cloud computing offers reductions in system administration, provisioning expenses, energy costs, software licensing fees, and hardware costs [12]

\section{CLOUD MIGRATION RISKS}

Many cloud migration challenges for the public or private sectors relate to its apparent newness and the relative underdevelopment of the marketplace for cloud services. The decisions to adopt cloud computing will be driven by more than technical and cost considerations. [12] Information is the lifeblood of organizations, and decisions on how to manage that information can have far-reaching political, social, and economic considerations. Adoption of cloud computing presents many of the same risks and challenges as deciding to use a more traditional outsourcing arrangement. The increased possibility that the service provider may reside outside of organizations legal or territorial jurisdiction, however, can make some of these issues more acute.

\subsection{Loss of Governance}

Users implicitly trust the service provider not to violate terms and conditions, and be in a position to securely store their information [13]. The terms and conditions set by the service provider may not actual conform with the established policies set by the consumers organisation. Non-compliance with such policies could lead to a loss of reputation and credibility. Service providers will also adapt their terms and conditions over time and often not inform users of these changes in an explicit manner. This presents the user with a dilemma: Either they accept terms that are not absolute or a privacy policy that they disagree with, or they do not use the service. Users may be unaware that the terms and conditions have changed and will use a service governed by policies and agreements which were not the ones originally agreed upon. Even more so, the user may be subject to peer pressure in which the service is also used by the users peer group and used actively for collaboration. Leaving the service may have an adverse effect 
upon the users interaction with their peers. Further problems arise when the users themselves fail to adhere to the conditions set out in the agreement e.g. failure to pay or the uploading of inappropriate material. What happens to the users data when this occurs? Will the data be retained and can it be extracted from the service provider? Furthermore a CSP will also change, over time, the terms and conditions attached to the service to further the CSPs own business interests [10]. Users are not in full control over the security of their data and that the protection offered by the service provider is not absolute.

\subsection{Lock-in}

Lack of cloud computing standards to promote a common way of developing and migrating applications to cloud that could guarantee portability and interoperability between applications and services and between vendors is another hurdle. This will result in forcing the client to be fully dependent on the service provider.[9] Enterprises adapt the standard offered by their vendors and get in to the risk of lock-in with a specific vendor thus giving away economies of multi-vendor models. In addition, there are very few vendors who offer full range of cloud computing capabilities pushing enterprises to adopt different cloud services from different vendors each using different standards. Level of lock-in might differ depending on what slice of cloud computing paradigm an enterprise is getting in to - Infrastructure-as- a-service providers like Amazon have low lock-in because of welldefined infrastructure standards and pretty common implementation by other IaaS providers, but platform and application providers like salesforce.com have high lock-in.

\subsection{Legacy Applications}

A big challenge for large enterprises planning to move their applications to cloud is the fact that their business processes are aligned with legacy systems which are decades behind in terms or technology and performance. Linking legacy systems with related applications in cloud pose integration and performance challenges, to take true advantage of cloud, legacy applications need to be rewritten. For most enterprises rewriting the legacy applications will be a multi-year long process which will result in parallel systems and business processes between traditional and cloud platforms, and that can become very problematic.

\section{CLOUD MIGRATION METHODOLOGY}

Once an enterprise has made decision to migrate to cloud, what are the frameworks they should follow? What qualifications criteria they should look for cloud migration, what applications or areas of their business they should move to cloud, what areas they should leave on-premise for now and how they should define their short and long term virtualization strategy. Migrating to the cloud brings lot of opportunities but different enterprises and industry domains will have their unique drivers and constrains for virtualization adoption. To answer these questions and many others we propose a methodology that enterprises can adopt to make a smooth transition to cloud environment. The proposed methodology is named Risk Homeostatic Methodology for Migrating to Cloud illustrated in figure 2. We propose a generic methodology which can be adopted and customized by enterprises to make a case for or against migrating to cloud. Listed below is basic representation and can be peeled down further for specific scenarios or industry requirements.

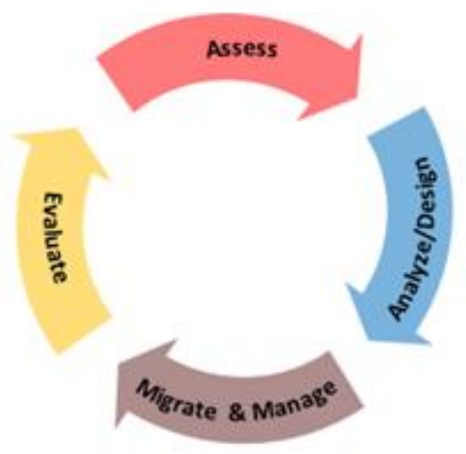

Figure 2: Risk Homeostas is Methodology for Migrating to Cloud

The outcomes of the following methodology are: should we migrate to cloud or not, what should we move, when should we move. Given that enterprise has made the decision to migrate to virtual environment, this methodology guides on how to migrate to cloud environment - what are the current opportunities in cloud environment, what architectures are available, which applications are good candidates for which cloud architecture? Each phase of the methodology contains a number of processes. Most processes can happen simultaneously (depending on the assessment resources) and the output of one can be the input of another, or the output of one might change the input of another and vice versa. The methodology, once applied to a system should never come to an end, as constant attention is needed to ensure that countermeasures remain appropriate and effective. The ultimate goal of this methodology is to help the enterprises to decide if they migrate to cloud or not and what they should move to cloud. The four phases of the methodology contains the following processes and activities:
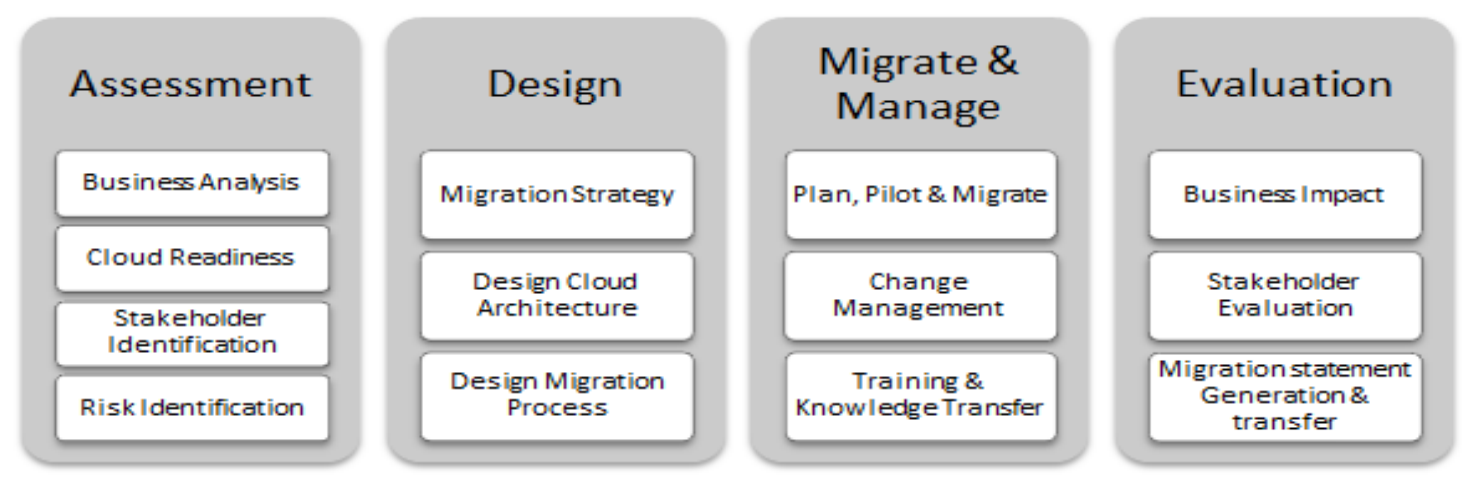

Figure 3: Risk Homeostasis Methodology Process 
1) ASSESSMENT: The very first step before acting on perhaps a big initiative like moving on-premise applications to Cloud would be the assessment of the organisation. The assessment will include the metrics indicating business analysis, cloud readiness, risk identification and key stakeholders which will give clear reason to move (or not) to cloud. An important part of this assessment phase will be setting of a baseline for assessing the impact of the investigation and estimating high level costs and resource requirements. This involves identifying key stakeholders to identify business needs. It also includes examining the costs, security and compliance, and identifies the gaps between our current traditional architecture and next-generation cloud architecture. Weighing the financial considerations of owning and operating a data center or co-located facilities versus employing a Cloud-based infrastructure requires detailed and careful analysis. The next process is combining and correlating information about assets and their use in support of the business. It baselines the environment and begins the process of asset classification. Each asset must be identified, and the portfolio of information regarding its use and interrelationship to the whole environment must be established and documented. The last process is to analyse and understand the risks, threats (and likelihood of those threats), and then based on sensitivity of our data, classify the information assets into different categories. This will help us to identify which datasets to move to the cloud and which ones to keep in-house

2) Design: Once the enterprise assessment is in place the next steps would be to design cloud computing migration strategy by aligning the investigation with the business strategy, and show how it can deliver business value. Show how the investigation might lead to changes that will affect business and the IT architecture environment. An important part of the Strategy will be to work with key stakeholders to identify business needs. In this phase, we evaluate which cloud computing models, architectures, technologies and best practices will make sense to implement (e.g., Private, Public or Hybrid Cloud) in your enterprise setting. Also assess the budgetary, resource and technical requirements necessary to prepare the business for pilot/testing phase of investigation. From financial perspective develop a total cost of ownership analysis and review established policies for assessing risks and change management.

3) Migrate and Manage: Once we have identified the right candidate for the virtual environment and estimated the efforts required to migrate. It is time to test the waters with a small proof of concept. The goal of this phase is to learn about the technology features and ensure that our assumptions regarding suitability for migration to the cloud are accurate. In this phase, the assessor can deploy a group of systems and, in the process, begin to get his feet wet with the cloud provider. Build a proof-of-concept that represents a microcosm of our virtual environment, or which tests critical functionality of the current systems in the virtual environment.[23] In this stage, we can build support in our organization, validate the technology, test legacy software in the cloud, perform necessary benchmarks and set expectations. After this stage, the assessor will have better visibility into what is available with virtualization providers; make careful assessment on the appropriate technology. The new environment will give the assessor more insight into what hurdles need to be overcome in order to move ahead.
4) Evaluation: In this phase, we evaluate the outcome of each phase and make decision for or against migration. This will give the organisation to understand cloud trends and evaluate what are their possible impacts on business objective, strategies and processes. Evaluation will indicate what should change - and what needs do not change and a tentative time frame for those changes. This step gives the management to have an easier job to decide how to spend money, by realizing the cost of each threat. Stakeholders will be actively involved in the assessment; hence it will be easier for them to understand the outcome. Personnel from different levels of the enterprise will be actively involved in the various processes of the methodology; hence through the proposed methodology, staff will achieve a level of education about the threats that they have to face in their corporate lives, otherwise unattainable.

\section{CONCLUSION}

This paper has explored different types of virtualization, cloud technologies and how can be used to improve resource management, simplify deployment, and improving the resilience of modern data centers. While the virtualization promises several benefits, migration to the cloud needs to be meticulously planned. Depending on business and technical considerations, organizations need to select the appropriate systems and infrastructure for migration, as all applications and infrastructure that are not suited for migration. A migration methodology is required to move systems to the virtual environment while navigating through various minefields.

This would enable organizations to ensure that the costs of migration to the virtual environment do not exceed its benefits. We have proposed a migration methodology that will guide organizations to move their on-premise datacenters to cloud. This methodology proposes specific steps which enterprises can adopt, both from business and technology perspective to make sure the migration and integration between on-premise and cloud happens with minimal disruption to business and results in maximum sustainable cost benefit.

To summarize, cloud computing holds promise for all types of organizations. However, different approaches are required by each organization to leverage the migration effectively. The methodology described here can be further enhanced and used by enterprises for a phased migration.

\section{REFERENCES}

[1] Cearley, D. W. Cloud Computing Key Initiative Overview. Gartner Research (2010).

[2] David S.Linthicum, Cloud Computing and SOA Convergence in Your Enterprise. Addison-Wesley Information Technology Series, 2010

[3] Janis Stirna, Anne Persson. Ten Years Plus with EKD: Reflections from Using an Enterprise Modeling Method in Practice. http://ceur-ws.org/Vol-365/paper10.pdf

[4] Rajkumar Buyya, Chee Shin Yeo, Srikumar Venugopal, James Broberg, Ivona Brandic, Cloud computing and emerging IT platforms: Vision, hype, and reality for delivering computing as the $5^{\text {th }}$ utility, Future Generation Computer Systems, Volume 25, Issue 6, June 2009, Pages 599-616, ISSN 0167 739X,10.1016/j.future.2008.12.001 
[5] Fehling, Christoph; Ewald, Thilo; Leymann, Frank; Pauly, Michael; Rtschlin, Jochen; Schumm, David, Capturing Cloud Computing Knowledge and Experience in Patterns,

http://www.iaas.unistuttgart.de/institut/mitarbeiter/fehlin g/INPROC-2012-104

[6] Luis, M. V., Luis, R,-M., et al. (2008) A break in the clouds: towards a cloud definition. SIGCOMM Comput. Commun. Rev., 39, 50-55.

[7] Cattedddu, D. and Hogben, G. (2009): Cloud Computing: Benefits, risks and recomendations for information security; European Network and Information Security Agency ENISA;

[8] jeffrey, k. and neidecker-lutz, b. (2009): the future of cloud computing: opportunities for European cloud computing beyond 2010; 66

[9] CHOW, R., GOLLE, P., et al. (2009) Cloud Computing: Outsourcing Computation Without Outsourcing Control. 1st ACM Cloud Comptuting Security Workshop. ACM

[10] JENSEN, M. and SCHWENK, J. (2009) On Technical Security

Issues in Cloud Computing. 2009 IEEE Conference on Cloud Computing. IEEE Computer Society

[11] Michael Armbrust, Armando Fox. Above the Clouds: A Berkeley View of Cloud Computing. Technical Report, February 10, 2009

[12] Dr Achrin Luhn, Michael Jaekel. Cloud Computing Business Models, Value Creation Dynamics and Advantages for Customers Dr Achrin Luhn, Michael Jaekel. Cloud Computing Business Models, Value Creation Dynamics and Advantages for Customers

[13] Microsoft Research, Securing Microsoft's Cloud Infrastructure," White Paper, 2009, http://www.globalfoundationservices.com/security/docu ments/SecuringtheMSCloudMay09.pdf

[14] Gary Anthesm, Security in the Cloud (2010) Communication of the ACM Vol. 53 No.11 [14] Ronald L. Krutz and Russell Dean Vines, Cloud Security, A comprehensive guide to Secure Computing

[15] Youseff, L., Butrico, M. and Da Silva, D. 2008. Toward a Unified Ontology of Cloud Computing. In Grid Computing Environments Workshop (GCE '08), Austin, Texas, USA, November 2008, 1-10.

[16] Sun Microsystems. 2009. Introduction to Cloud Computing architecture. Whitepaper. Sun Microsystems.

[17] Luis Vaquero, Luis Rodero-Merino, Juan Caceres, et al, "A break in the clouds: towards a cloud definition,"
ACM SIGCOMM Computer Communication Review, vol. 39, pp. 50-55, 2009.

[18] Gary Anthesm, Security in the Cloud (2010) Communication of the ACM Vol. 53 No.11

[19] Virtualization from the Datacenter to the Desktop. (2007). White paper, Microsoft virtualization Resources.

[20] The Cloud :Changing the business ecosystem,2011 www.kpmg.com

[21] Seccombe A, Hutton A, Meisel A, Windel A, Mohammed A, Licciardi A, et al. Security guidance for critical areas of focus in cloud computing, v2.1. Cloud Security Alliance, 2009, 76p

[22] NIST, NIST Definition of cloud omputing v15, NIST, Editor. 2009, National Institute of Standards and Technology: Gaithersburg, MD (2009).

[23] A. Khajeh-Hosseini, I. Sommerville, and D.Greenwood, Cloud Migration: A Case Study of Migrating an Enterprise IT System to IaaS Submitted to 1st ACM Symposium on Cloud Computing (SOCC 2010), 2010

[24] Sotto, L. J., Treacy, B. C., et al, Privacy and Data Security Risks in Cloud Computing; The National Bureau of National Affairs, Inc; 15 ECLR 186. (2010)

[25] LSE, Castro, D, Grous, A , Karrberg, P, Modelling the Cloud - Employment effects in two exemplary sectors in The United States, the United Kingdom, Germany and Italy, January 2012

[26] Redshift Research, Adoption, Approaches and Attitudes:The Future of Cloud Computing in the Public and Private Sectors, 2011

[27] D.C. Wyld, Moving to the cloud: An introduction to cloud computing in government. Washington, DC: IBM Center for the Business of Government, November 2009.

[28] Hustinx, P., Data Protection and Cloud Computing under EU law, Third European Cyber Security Awareness Day, BSA,European Parliament 2010.

[29] Xuan Zhang Wuwong, N. Hao Li Xuejie Zhang Information Security Risk Management Framework for the Cloud Computing Environments in 2010 IEEE 10th The International Conference Computer and Information Technology (CIT), 2010,pp. 13281334.

[30] Pearson, S. and Azzedine Benameur, Privacy, Security and Trust Issues Arising from Cloud Computing in 2010 IEEE Second International Conference Cloud Computing Technology and Science (CloudCom),Nov 30-Dec 3,2010, page(s): 693- 702 . 